\title{
O STATUS CIENTÍFICO DA PESQUISA EM ADMINISTRAÇÃO ${ }^{1}$
}

\author{
THE SCIENTIFIC STATUS OF MANAGEMENT RESEARCH
}

\author{
Silvana Anita Walter ${ }^{2}$ \\ Paulo Otávio Mussi Augusto ${ }^{3}$
}

\begin{abstract}
RESUMO: O presente artigo parte da idéia de que a administração tem tido dificuldades para ser caracterizada como ciência. O debate existente sobre esta temática, entretanto, não chegou a um acordo sobre quais critérios de cientificidade poderiam guiar esta área de conhecimento. Este ensaio procura preencher esta lacuna teórica ao destacar os principais motivos pelos quais a administração encontra dificuldades para se consolidar como ciência, ao discutir o status científico da pesquisa em administração e, ao mesmo tempo, procurar responder ao questionamento sobre quais critérios esta deve buscar atender para alcançar status de ciência. A pesquisa bibliográfica realizada permitiu encontrar dois principais motivos para as dificuldades de classificação da administração como ciência: (a) a administração, como ciência social, não atinge alguns dos critérios de cientificidade apregoados pelas ciências naturais e (b) a validade e a relevância dos resultados obtidos pelos estudos em administração. Após esta discussão, argumenta-se que a cientificidade da administração não deve ser julgada com base no padrão das ciências naturais, visto que estas ciências são diferentes e uma não poder ser substituída pela outra. Observa-se, também, que, para alguns estudos, as pesquisas em administração necessitam ampliar seu rigor metodológico e apresentar contribuições importantes para as práticas de gestão das organizações. Ressalta-se que a administração, para alcançar o status de ciência, deve atingir os critérios de validade e relevância, como também desconsiderar os critérios de cientificidade das ciências naturais, visto que estes não se aplicam a ela. Diante dos aspectos apontados e discutidos neste ensaio, defende-se a posição de que a administração pode gerar conhecimento científico, necessitando, porém, independente da posição epistemológica, zelar mais pelo rigor metodológico dos estudos da área, para que seus resultados sejam válidos, bem como buscar contribuições relevantes que melhorem a gestão das organizações, visto que possui uma natureza aplicada.
\end{abstract}

Palavras-chave: Status científico. Ciências sociais. Administração.

ABSTRACT: The present article is based on the idea that the discipline of Management finds difficulties to consolidate itself as science. The current debate about this issue doesn't have reached a consensus about what criteria we should use to show the scientific status of the discipline. This essay seeks to fulfill this gap by discussing the main issues that create difficulties for management establish itself as a science. Based on a bibliographic research two reasons are pointed out for that. The first concerns to the fact that management, as social science, does not reach any of the scientificity criteria reached by the natural sciences, which occurs due to the complexity of the its object of study and the fact that social and natural sciences are distinct and its not possible the replacement of one by another, making inconsistent to judge management by the natural sciences criteria. The second reason is related to the validity and relevance of the results obtained by management research. In face of the highlighted and discussed aspects in this essay it is defended that Management doesn't should use the same scientific criteria of the natural sciences. It is observed that the researches require expanding their methodological rigor and present relevant contributions to the organizations management. Finaly, it is defended the position that management can generate scientific knowledge, requiring, however, independently of epistemological position, to take more care of methodological rigor of studies in the area, so that their results are valid, as well to seek relevant contributions that improve the organizations management, considering that management has an applied nature.

Key-words: Scientific status. Social sciences. Management.

\footnotetext{
${ }^{1}$ Artigo Recebido em 08.07.2008 . Revisado por pares em 18.03.2009 . Recomendado em 18.03.2009 por Denise Del Pra Netto Machado (editora). Publicado em 22.04.2009.

Organização Responsável pelo periódico: Universidade regional de Blumenau - FURB - www.furb.br/rn
}

\footnotetext{
${ }^{2}$ Pontifícia Universidade Católica do Paraná - PUCPR - silvanaanita.walter@gmail.com

${ }^{3}$ Pontifícia Universidade Católica do Paraná - PUCPR - paulo.augusto@pucpr.br
} 


\section{INTRODUÇÃO}

Desde a sistematização dos conhecimentos sobre administração, o meio acadêmico vem discutindo se esta pode ser considerada uma ciência. Neste sentido, Granger (1994) indica que alguns cientistas consideram errônea a atribuição do termo ciência ao conhecimento dos fatos humanos, por estes não possuírem a fecundidade e a solidez dos saberes biológicos, químicos e físicos. Entretanto, Thompson (1956) assevera que a conceituação da administração como ciência deve ser seriamente considerada.

Argumenta-se, neste ensaio, que a administração tem dificuldade para ser considerada ciência em virtude de dois motivos: o primeiro consiste no fato de esta não atingir alguns dos critérios de cientificidade alcançados pelas ciências naturais, o que é compartilhado por todas as ciências sociais, e o segundo refere-se à validade e à relevância dos resultados obtidos pelos estudos em administração.

Em referência aos critérios de cientificidade, tem-se que, para Whitley (1977), a administração pode ser classificada como ciência social, visto que seu objeto de estudo se constitui de fenômenos de ordem social, ou seja, do estudo e da melhoria da coordenação e do controle de atividades humanas associadas. Pretender ser uma ciência social implica, segundo o padrão atual de ciência baseado nas ciências naturais, em dificuldades para a sua caracterização como ciência. Em relação ao mesmo motivo, tem-se, também, de acordo com Basso (2004), que o fato de as ciências sociais não conseguirem atingir a neutralidade e a objetividade das ciências naturais contribui para manter a suposta "superioridade" das ciências naturais em detrimento das ciências sociais.

A dificuldade que as ciências sociais, em geral, e a administração, em particular, encontram para atingir a neutralidade e a objetividade que possuem as ciências naturais devese à complexidade de seu objeto de estudo, como mencionam Granger (1994) e Lévi-Strauss (1976). Isso porque os fenômenos de comportamento humano possuem significações que vão contra sua transformação em esquemas lógicos e matemáticos (GRANGER, 1994), como ocorre nas ciências naturais.

No que tange à validade dos resultados obtidos pelos estudos em administração, sabese que os estudos, para serem válidos, necessitam, independentemente do posicionamento epistemológico adotado, de um amplo rigor metodológico em seu desenvolvimento e apresentação final. Já sobre a relevância dos resultados dos estudos em administração, esta, enquanto uma ciência aplicada (THOMSON, 1956), deveria gerar conhecimentos aplicáveis e importantes para a melhoria dos processos de gestão das organizações.

Estudos anteriores já analisaram a conceitualização da administração ou dos estudos sociais como ciência, como o caso de Machlup (1994) e Silva (2002). Machlup (1994) analisa as limitações das ciências sociais em relação às ciências naturais e Silva (2002) explora se a administração é ou não ciência. Assim, não foram encontrados estudos que explorassem os motivos pelos quais a administração encontra dificuldades para se consolidar como ciência e quais os caminhos a serem seguidos para buscar essa consolidação, portanto esse estudo procurará preencher essa lacuna teórica.

Diante do contexto apresentado, este ensaio tem por objetivo discutir o status científico da pesquisa em administração, ou seja, a possibilidade de conceitualização da administração como ciência. Neste sentido, procurar-se-á responder à seguinte pergunta de pesquisa: Quais critérios de cientificidade a administração necessita seguir para que alcance status de ciência? Para tanto, faz-se, na seção 2, uma discussão sobre os critérios utilizados para atribuição de uma suposta "superioridade" às ciências naturais em detrimento das sociais, como a administração. Na seqüência, na seção 3, debate-se a conduta metodológica dos estudos em administração no que se refere à validade e à relevância e, na seção 4, procura-se discutir a possibilidade de a administração ser considerada uma ciência. 


\section{CRITÉRIOS DE CIENTIFICIDADE}

Conforme já mencionado, o primeiro motivo pelo qual a administração não é, algumas vezes, considerada uma ciência refere-se a sua dificuldade de atingir os critérios de cientificidade das ciências naturais.

A diferença entre ciências naturais e sociais ocorre, porque, conforme destaca Whitley (1984), o mundo social é essencialmente diferente do mundo natural inanimado; o conhecimento resultante das pesquisas é obtido de modo particular; e, as descrições dos atores são prioritárias sobre a descrição feita pelos pesquisadores.

Na direção indicada, a dificuldade que as ciências sociais encontram para atingir os critérios de cientificidade alcançados pelas ciências naturais é fruto de seu objeto de estudo complexo - que consiste nas experiências cotidianas que os indivíduos partilham em sociedade (SCHUTZ, 1979) -, visto que essas relações sociais passam por um processo constante de mudança histórica e cujo núcleo é o indivíduo dotado de certa liberdade, singularidade e imprevisibilidade.

Assim, essa dificuldade de aceitação das ciências sociais como ciência por parte da academia afeta, conseqüentemente, a administração, uma vez que as organizações, enquanto entidades sociais formadas por indivíduos, também possuem certa imprevisibilidade e passam por mudanças históricas. Dessa forma, por ser uma ciência social, a administração é, por vezes, considerada inferior às ciências naturais no que tange ao caráter científico.

Entre as características utilizadas para justificar a suposta "superioridade" das ciências naturais em detrimento das ciências sociais e, em conseqüência, da administração, Machlup (1994) questiona a variabilidade dos fenômenos, a subjetividade envolvida, a dificuldade de verificar hipóteses, a falta de exatidão nos resultados, a dificuldade de mensurar os fenômenos e a dificuldade de prever eventos futuros.

Na natureza, existem mais fatores e condições invariáveis do que na sociedade. Essa variabilidade nos fenômenos sociais provoca dificuldade de generalização dos resultados encontrados nesses estudos. Todavia, os fenômenos sociais não são tão variáveis quanto se pode imaginar, pois, se assim o fossem, nem mesmo seria possível generalizar os conceitos e termos empregados para nomear esses fenômenos (MACHLUP, 1994).

Thompson (1956) destaca que o entendimento de que os comportamentos humanos não possuem regularidades é equivocada e reflete a falta de habilidade de teóricos em encontrar as regularidades do comportamento humano. Argumenta esse autor que todo o ensino e o treinamento para que as pessoas exerçam funções administrativas estão fundamentados nas regularidades dos comportamentos envolvidos; caso contrário, se toda ação administrativa fosse única, não haveria conhecimento a ser transferido. Assim, como não é possível negar a existência do ensino em administração, Thompson (1956) confirma a existência de regularidades nos comportamentos humanos estudados em administração.

Nesse mesmo sentido, Tsoukas e Cummings (1997) mencionam que a ciência administrativa é considerada possível porque, atrás da variedade aparente das organizações, há uma ordem que pode ser capturada pelos cientistas sociais por meio de seus métodos de investigação.

No que tange à subjetividade, as ciências naturais procuram impor às ciências sociais seu ideal de objetividade (BASSO, 2004). Sobre esse ideal, acredita-se que a ciência não deveria realizar julgamentos de valor. Nesse sentido, a administração e as ciências sociais sofreriam de subjetividade, uma vez que as observações das ações humanas só podem ser interpretadas por meio dos propósitos e motivos dos atores, ou seja, por valores (MACHLUP, 1994). Essa distinção entre as ciências sociais e naturais ocorre, segundo Machlup (1994), porque, para explicar os caminhos dos corpos celestes ou o movimento das moléculas, um cientista natural não questionará por que tal planeta escolheu essa órbita ou por que as 
moléculas querem se mover. Já o cientista social, segundo o mesmo autor, precisará compreender por que determinado fenômeno está acontecendo, uma vez que fenômenos sociais são resultado de ações humanas incentivadas. Assim, é imprescindível que os cientistas sociais interpretem os fenômenos quanto aos motivos e propósitos dos atores.

Lévi-Strauss (1976) indica que o subjetivismo envolvido nos estudos sociais ocorre porque o indivíduo não poderia observar de forma objetiva um fenômeno, já que está incluído no próprio contexto, abrangendo, assim, seu interesse pessoal. Astley (1985) corrobora essa idéia e considera a ciência administrativa uma construção social, moldada pelos pontos de vista subjetivos, por meio dos quais a realidade é construída.

No contexto apresentado, poder-se-ia dizer que a administração e as ciências sociais podem sofrer com a influência de visões e conceitos particulares do pesquisador nos resultados encontrados. Porém, pode-se perceber a ocorrência dessa mesma dificuldade também nas ciências naturais, como o fato de teorias sobre a evolução, por exemplo, não serem aceitas por contrariarem preceitos religiosos ou o fato de matemáticos e físicos alemães terem rejeitado teorias de pesquisadores judeus (MACHLUP, 1994). Outro exemplo a ser destacado é o de Galileu, porquanto, ao criar sua teoria para explicar o movimento das marés, desconsiderou a influência lunar, já destacada anteriormente por Kepler. Segundo Popper (1975), um dos motivos pelos quais Galileu rejeitou a influência lunar em sua teoria foi que este era contrário à astronomia e adversário de Kepler. O motivo apontado pelo autor deixa perceptível que uma pré-concepção de Galileu a respeito da astronomia e de Kepler fez com que desconsiderasse um importante fator de influência nas marés.

Percebe-se que há possibilidade de preconceitos ou conceitos previamente elaborados influenciarem a apresentação de evidências e de resultados, bem como a aceitação destes afetarem tanto as ciências sociais quanto as ciências naturais. A diferença, no que se refere à forma de interpretação dos fenômenos nas ciências sociais e naturais, é conseqüência de distinções em relação a características de cada ciência. Assim, como destaca Weber (1947), o propósito das ciências físicas é "explicar” e o das ciências humanas é “entender”.

Em referência à verificabilidade de hipóteses, Machlup (1994, p. 10) destaca que "a impossibilidade de experimentos controlados e o número relativamente grande de variáveis pertinentes são os obstáculos principais para a verificação mais eficiente nas ciências sociais”. Isso ocorre porque as ciências sociais não podem isolar a sociedade e seus indivíduos em um laboratório para estudá-los. Assim, a dificuldade de verificar hipóteses, nas ciências sociais e na administração, se deve à complexidade do próprio objeto observado. No entanto, sabe-se que, mesmo em experimentos ou em observações utilizados nas ciências naturais, nem sempre é possível assegurar que fatores não observados possam interferir nos resultados.

Para amenizar a dificuldade que a administração e outras ciências sociais possuem para saber se existem ou não outros fatores não considerados interferindo nos efeitos observados, têm-se o uso do critério de validade interna. Esta, de acordo com Straub e Carlson (1989), avalia se os efeitos observados poderiam ter sido causados por um conjunto de variáveis não hipotéticas e/ou imensuráveis.

A exatidão nos resultados, segundo Machlup (1994, p. 11), refere-se à construção de "um sistema teórico de modelos idealizados que contêm variáveis ou construtos abstratos e de relações entre variáveis, das quais podem ser deduzidas a maioria ou todas as proposições relativas a conexões particulares”. Para esse autor, a exatidão não pode ser atribuída nem mesmo a muitas das ciências naturais, caso de algumas áreas da biologia, por exemplo.

A mensurabilidade de fenômenos está relacionada, para Machlup (1994), à quantificação de dados, possivelmente porque esse autor dá ênfase à economia dentro das ciências sociais. Conforme o autor ressalta, não é possível saber onde existem mais fatores que não podem ser quantificados: na natureza ou na sociedade. Não obstante, resta a pergunta: até que ponto a quantificação significa mensuração? Ou existem fenômenos que podem ser 
mais bem entendidos sem a utilização da lógica matemática? Diante da complexidade dos fenômenos estudados na administração, em que medida a quantificação de dados permite respeitar essa complexidade?

O emprego do critério de quantificação para verificar a mensurabilidade dos fenômenos parece ir ao encontro dos critérios propostos pelas ciências naturais e, como já destacado, não atingidos por todas elas, uma vez que costumam se apresentar como exatas. Esse pensamento ratifica o ressaltado por Basso (2004) de que as ciências naturais procuram impor seu método quantitativo às ciências sociais. Resta saber se a administração e as demais ciências sociais desejam que sua mensurabilidade seja avaliada apenas por meio da quantificação, bem como se ela respeita a complexidade de seu objeto de estudo.

Costuma-se apontar que a administração, assim como as demais ciências sociais, possui dificuldade em prever eventos futuros. Isso está relacionado às mudanças costumeiramente ocorridas na sociedade e nas organizações, à imprevisibilidade dos indivíduos pertencentes a elas e à variabilidade dos fenômenos. Este fato, contudo, não afeta apenas as demais ciências sociais, pois, afinal, cientistas naturais, como físicos e engenheiros, também têm dificuldade para prever o comportamento de máquinas novas, ainda não testadas (MACHLUP, 1994). Dessa forma, diante das características de individualidade dos atores da sociedade e das organizações, cada ator pode ser considerado novo, visto que é diferente dos outros já estudados. Entretanto, mesmo diante desse contexto, a administração pode se concentrar em generalizações mais abstratas que possam antecipar comportamentos futuros.

Essa dificuldade das ciências sociais atingirem cientificidade, conforme expõe Granger (1994), também está relacionada à sua relação com a história. Para esse autor, as ciências dos fatos humanos encontram problemas na validação de seus enunciados por dois aspectos: os fatos são estabelecidos por meio de vestígios materiais e do testemunho de pessoas ou documentos, e, a dificuldade de validação das explicações históricas, pois estas não podem se alicerçar na repetição de fenômenos. Nesta direção, Machlup (1994) destaca que isso também ocorre nas ciências naturais quando cientistas naturais testemunham como peritos em tribunais, analisando um evento individual da história recente.

Lévi-Stauss (1976) ressalta que outro aspecto importante para uma ciência é a obtenção de um método homogêneo. Contudo, observa-se que a administração e as ciências sociais não atingiram um consenso em relação a um único método. Isso porque há uma divisão, nessas ciências, no que concerne à utilização desse método, como ocorre, por exemplo, nos métodos propostos pelo paradigma funcionalista ou pelo interpretativista.

O paradigma funcionalista busca prover explicações essencialmente racionais de fenômenos sociais, preocupa-se em entender a sociedade gerando conhecimento utilizável e em prover soluções práticas para problemas práticos. Está arraigado no positivismo sociológico, refletindo a tentativa de aplicar os modelos e os métodos das ciências naturais ao estudo dos fenômenos sociais (HATCH; YANOW, 2005).

Já o paradigma interpretativo preocupa-se em entender o mundo social no âmbito da experiência subjetiva e busca as explicações na consciência individual (BURREL; MORGAN, 2000). As filosofias interpretativas, conforme Hatch e Yanow (2005), se desenvolveram contra os positivistas lógicos e apontavam que a pesquisa interpretativa não poderia utilizar os padrões e os métodos normativos da pesquisa positivista. Sob essa perspectiva, Hatch e Yanow (2005) consideram que as realidades sociais são construídas pelos sujeitos que agem em conjunto, sendo assim distintos o mundo social e o mundo natural. Diante dessa distinção, os interpretativistas argumentam que os atos sociais somente serão compreendidos por meio da interpretação e que a interpretação de atos, da linguagem e de objetos é o único meio para estudar o mundo humano e social (HATCH; YANOW, 2005).

Percebe-se uma divergência na administração e nas ciências sociais em relação ao paradigma de referência. Dijk e Punch (1993) destacam que a ciência administrativa é 
desenvolvida, majoritariamente, por meio de métodos quantitativos e terminologia de ciência natural, assim como Machado-da-Silva, Cunha e Amboni (1990) salientam que 80,3\% dos trabalhos em administração, no Brasil, concentram-se no paradigma funcionalista.

Já Astley (1985) e Hatch e Yanow (2005) discordam da perspectiva funcionalista e sustentam uma metodologia diferenciada para a administração: a interpretativa. Nesse sentido, Hatch e Yanow (2005) defendem que as ciências que tratam de fenômenos humanos e sociais precisam de um método distinto para a compreensão desses fenômenos. Indo ao encontro desta perspectiva, Habermas (1968) destaca que, por meio da assimilação dos métodos das ciências naturais, as ciências sociais tornaram-se “cientísticas”, que, segundo Ramos (1989), significa que a compreensão da realidade, para ser correta, deve ser descrita em consonância com a linguagem técnica das ciências naturais; todavia, este autor critica essa concepção.

Observa-se, por meio dos aspectos destacados, que a administração e as ciências sociais não atingiram um consenso em relação a um único método para suas pesquisas. Contudo, constata-se, por meio do histórico das correntes epistemológicas e metodológicas no desenvolvimento da ciência, que esse consenso inicialmente também não existia em outras áreas, a exemplo das ciências naturais e de sua disputa entre métodos de indução e dedução.

Os argumentos aqui apresentados procuram demonstrar que os critérios utilizados para atribuir uma suposta "superioridade" às ciências naturais e para as tornarem parâmetro de ciência não se referem a falhas ou a defeitos das ciências sociais, mas a características do objeto estudado por estas, bem como que a suposta inferioridade atribuída à administração e às demais ciências sociais está fundamentada em critérios e na ótica das ciências naturais.

\section{VALIDADE E RELEVÂNCIA DOS ESTUDOS EM ADMINISTRAÇÃO}

Conforme destacado, o segundo motivo pelo qual a administração encontra dificuldades para ser considerada ciência refere-se à validade e à relevância dos resultados obtidos pelos estudos da área.

A produção acadêmica em administração, no mundo todo e, especialmente, no Brasil, está em crescimento, o que se pode confirmar pela observação do crescimento no número de estudos submetidos e publicados em eventos e periódicos da área, bem como no número de dissertações e teses desenvolvidas. Porém, esse crescimento quantitativo, na visão de Bertero, Caldas e Wood Jr. (1999), não é proporcional ao crescimento qualitativo das mesmas.

Definir critérios de qualidade para a realização de estudos é uma tarefa complexa. No entanto, a validade e a relevância dos resultados obtidos nos estudos são de fundamental importância, fazendo-se necessárias a adoção de procedimentos metodológicos rigorosos e a busca por contribuições relevantes para a gestão das organizações.

Entre os fatores de fundamental importância para o rigor metodológico de um estudo, se encontra a validação da pesquisa, que, de acordo com Straub e Carlson (1989), é extremamente relevante, pois, sem ela, os resultados dos estudos não são confiáveis. Além disso, a comparação constante entre a teoria e a prática no processo de validação resulta em variáveis teoricamente mais significantes (BAGOZZI, 1980).

Porém, estudos para avaliar a validade de medidas são relativamente raros porque seus desenhos de pesquisa são, normalmente, complexos e porque a maioria dos pesquisadores sociais prefere construir e testar teorias ao invés de conferir a precisão dos seus instrumentos de mensuração (BREWER; HUNTER, 2006).

Straub e Carlson (1989) revisaram estudos da área de administração de sistemas de informação (MIS) publicados em periódicos norte-americanos e constataram que 62\% deles não empregavam nenhuma forma de validação; que a confiabilidade foi o critério de validade mais empregado, sendo que, no entanto, $83 \%$ dos estudos não a testavam; que a confiabilidade não costuma ser combinada com a validade de construto; e que a validade de 
conteúdo é praticamente desconhecida. Os autores observaram, ainda, que muitos estudos confiam em instrumentos já empregados em pesquisas anteriores. Quanto a isso, os autores sugerem cautela, visto que muitos desses instrumentos previamente utilizados nunca foram validados e que as alterações realizadas pelos pesquisadores no instrumento anteriormente validado para adaptá-lo à nova pesquisa, podem gerar um instrumento sem validade.

Além da validação das pesquisas realizadas em administração, outros aspectos relacionados ao rigor metodológico e à contribuição dos estudos são de vital importância para que essa área seja considerada uma ciência. No entanto, destacam-se algumas pesquisas que revelam que os estudos realizados possuem vários aspectos a serem aperfeiçoados.

Clark, Floyd e Wright (2006) identificaram como os principais responsáveis pela rejeição de artigos submetidos ao Journal of Management Studies, no período de 2003 a 2004: a falta de contribuição dos estudos, encontrada em 92\% dos estudos rejeitados; o fracasso em desenvolver contribuição teórica, presente em $76 \%$ das pesquisas rejeitadas; falhas fatais nos métodos, encontradas em $70 \%$ dos artigos rejeitados; e deficiências nas análises, presentes em $58 \%$ dos estudos rejeitados.

A falta de contribuição dos artigos, para Clark, Floyd e Wright (2006), refere-se ao fato de que a contribuição para a literatura existente na maioria dos estudos não é evidente ou é marginal. Isso ocorre, de acordo com os autores por três motivos. O primeiro, pelo fato de que os autores dos estudos freqüentemente não identificam, na literatura anterior, a lacuna particular que desejam preencher. $\mathrm{O}$ segundo motivo consiste em os estudos serem escritos de tal modo que várias contribuições potenciais são reivindicadas, mas com falta de clareza sobre o foco do documento. O terceiro refere-se à contribuição não ser recente.

Não apresentarem uma contribuição teórica ou não desenvolverem e testarem hipóteses novas que possam desenvolver ou ampliar o entendimento conceitual de um fenômeno é a segunda razão para a rejeição dos artigos, segundo Clark, Floyd e Wright (2006). De acordo com os autores, esses estudos são freqüentemente muitos descritivos e falham em fornecer explicações para as relações observadas entre as variáveis ou padrões presentes nos fenômenos descritos.

Em muitos estudos rejeitados, segundo Clark, Floyd e Wright (2006), o design da pesquisa é fundamentalmente falho ou impróprio para a questão da pesquisa escolhida. Para os autores, isto pode significar que: a estrutura da amostra é imprópria; os construtos e as medidas foram inadequadamente operacionalizados; as inter-relações entre os construtos não foram especificadas; e há divergência entre as medidas empregadas e as hipóteses ou o modelo. Em estudos quantitativos, Clark, Floyd e Wright (2006) destacam que os problemas particulares podem surgir ao serem empregados conjuntos de dados projetados para propósitos diferentes daqueles da apresentação ou ao serem reduzidos os dados tão sutilmente, fazendo com que isto se sobreponha a outros trabalhos publicados ou submetidos. Já no caso de estudos qualitativos, os autores ressaltam que estes também deveriam ser projetados cuidadosamente com uma pergunta teórica em mente. Neste sentido, Clark, Floyd e Wright (2006) ressaltam que uma das falhas mais comuns dos projetos de estudo qualitativos é o uso de um estudo de caso para ilustrar ou testar uma teoria estabelecida, pois normalmente se espera que percepções teóricas novas surjam da análise de dados qualitativos.

Por fim, Clark, Floyd e Wright (2006) citam que um trabalho pode ser rejeitado pela existência de problemas fundamentais na forma por meio da qual os dados são analisados. Isto se relaciona, segundo os autores, principalmente ao uso de métodos impróprios ou inadequados em relação à pergunta da pesquisa.

Pode-se notar que o terceiro e o quatro motivos apontados por Clark, Floyd e Wright (2006), estão intimamente interligados, sendo que poderiam ser, até mesmo, agrupados. Assim, ter-se-ia um percentual ainda maior de estudos que apresentaram essas limitações de falhas fatais nos métodos e deficiências nas análises. 
Hoppen e Meirelles (2005) realizaram um panorama da pesquisa científica em sistemas de informação publicada em eventos e periódicos brasileiros, sendo eles o Encontro da Associação Nacional de Pós-Graduação e Pesquisa em Administração (EnANPAD), a Revista de Administração da Universidade São Paulo (RAUSP), a Revista de Administração de Empresas (RAE), a Revista de Administração Pública (RAP), a Revista de Administração Contemporânea (RAC) e a revista Organização e Sociedade (O\&S). Os autores avaliaram, entre outros aspectos, a qualidade científica (metodológica) de artigos publicados no período de 1990-1997. Como resultado da avaliação da qualidade metodológica, Hoppen e Meirelles (2005) constaram que $26 \%$ dos estudos revisados não apresentam teorias e conceitos de base e 49\% as apresentam de forma superficial; 53,1\% não explicitam as questões ou hipóteses de pesquisa; $43,8 \%$ não apresentam o modelo de pesquisa e $27 \%$ o apresentam com pouca clareza; 37,5\% não apresentam recomendações e limites do estudo; 53,3\% das pesquisas do tipo survey e experimentais não explicitam a operacionalização das variáveis; $60 \%$ das pesquisas survey e experimentais não apresentam informações sobre a validade de face, $75,6 \%$ sobre a confiabilidade e $86,7 \%$ sobre a validade do construto; apenas $40 \%$ das pesquisas survey e experimentais realizaram a escolha da amostra de forma aleatória; apenas $17,1 \%$ das pesquisas survey combinam dados primários e secundários, bem como apenas 25,5\% das pesquisas qualitativas empregam múltiplos métodos para coleta de dados; $50 \%$ dos testes estatísticos realizados em pesquisas experimentais não são pertinentes; $30 \%$ das pesquisas experimentais não possuem um desenho experimental adequado nem um controle experimental rigoroso; 88,6\% das pesquisas qualitativas não têm o protocolo de análise dos dados explicitado; e, 43,1\% das pesquisas qualitativas possuem uma interpretação superficial dos dados.

Para Hoppen e Meirelles (2005), a qualidade científica classificada como "baixamédia” nos artigos científicos de pesquisas empíricas por eles revisados são conseqüência do pouco rigor no que se refere ao desenho de pesquisa e à validade dos instrumentos. Isso, segundo os autores, revela dois problemas: a necessidade de intensificar o rigor científico no desenvolvimento das pesquisas e a falta de informações importantes na apresentação nos artigos científicos. A explicitação precária do modo como as teorias de base foram operacionalizadas e a descrição incompleta dos procedimentos metodológicos são aspectos adicionais que comprometem a qualidade dos artigos (HOPPEN; MEIRELLES, 2005).

A partir das constatações a que chegaram, Hoppen e Meirelles (2005) apresentam orientações para a realização de estudos. Segundo os autores, quando se optar pela pesquisa do tipo survey, devem-se levar em consideração elementos como a definição da amostra e a validação dos instrumentos de pesquisa. Quando o método escolhido for o estudo de caso, são fundamentais elementos como a atenção com a escolha de casos - simples ou múltiplos - que levem em consideração fenômenos críticos - novos ou originais -, o rigor na definição das questões de pesquisa, o cuidado na descrição dos procedimentos de coleta e análise dos dados e a combinação de dados primários e secundários. Para os mesmos autores, também é fundamental, nos estudos de caso realizados segundo a abordagem positivista, a validação dos instrumentos de pesquisa e dos procedimentos de codificação dos dados. Em relação às pesquisas com métodos qualitativos, os autores ressaltam que os eventos e periódicos deveriam oferecer a possibilidade de um número de páginas maior do que os limites impostos às pesquisas de caráter quantitativo, permitindo-se, dessa forma, melhor apresentação dos procedimentos metodológicos adotados para a coleta e análise dos dados.

Walter, Baptista e Augusto (2008) revisaram estudos sobre Resourse Based Review (RBV) publicados, no período de 1997-2007, na área de estratégia do EnANPAD. Os autores constataram que os estudos revisados possuem diversos aspectos metodológicos que podem ser aperfeiçoados. Entre eles, pode-se mencionar: poucos estudos realizaram algum tipo de validação; 9,7\% dos estudos de caso não apresentam os instrumentos de coleta de dados 
empregados; os estudos teórico-empíricos que utilizam como instrumento de coleta de dados um questionário não citam a realização de um pré-teste ou de avaliação com especialistas da área; os levantamentos, em sua maioria, não descrevem de forma detalhada os questionários e os roteiros de entrevista utilizados, bem como os estudos de caso, as fontes bibliográficas, os meios de comunicação de massa, os documentos e os roteiros de entrevistas; apenas $25 \%$ dos levantamentos e 64,5\% dos estudos de caso realizam a combinação de dados primários e secundários; grande parte dos levantamentos utiliza amostras não-probabilísticas; 48,4\% dos estudos de caso e 6,3\% dos levantamentos não apresentam o método de análise utilizado; e um número relativamente considerável de estudos teórico-empíricos não apresenta uma descrição do método de pesquisa utilizado ou a apresenta de forma não muito clara.

Como aspectos positivos encontrados nos estudos revisados, Walter, Baptista e Augusto (2008) citam a utilização de métodos adequados aos objetivos propostos nos estudos; a coerência entre o tipo de estudo realizado e os métodos de coleta e análise de dados empregados; o fato de que a maioria dos artigos apresenta um objetivo definido para a pesquisa; e a existência de alguns ensaios teóricos que apresentam proposições teóricas.

Com base nos resultados encontrados, Walter, Baptista e Augusto (2008) propõem que os estudos teórico-empíricos tenham um maior rigor metodológico no que se refere à apresentação de informações no relatório final, como questão de pesquisa, hipóteses, desenho da pesquisa, detalhamento dos instrumentos de coleta de dados (no sentido de ser possível apresentar como apêndice ou anexo o instrumento utilizado, a exemplo de artigos internacionais) e método de análise dos dados. Além disso, os autores sugerem que os estudos teórico-empíricos façam uso, de forma coerente ao tipo de estudo e posição epistemológica, de critérios de validação das pesquisas; de diferentes fontes de dados; e de amostras probabilísticas que permitam maiores generalizações, quando embasados na perspectiva positivista. Em relação aos ensaios teóricos, os autores ressaltam que poderia ser ampliado o número de estudos que apresentam proposições teóricas.

Diante do apresentado, destaca-se a importância dos estudos em administração adotarem procedimentos metodológicos rigorosos, independente do tipo de estudo realizado ou da posição epistemológica adotada, bem como descreverem de forma detalhada os procedimentos adotados em relação ao desenho de pesquisa, à coleta e à análise de dados e à validação da pesquisa.

Bertero, Caldas e Wood Jr. (1999) encontraram outra limitação nas pesquisas realizadas na área de administração de empresas no Brasil: o tipo de revisão de literatura realizada. A respeito desse aspecto, Lima (1999) destaca que muitos referenciais teóricos expostos em artigos não configuram uma lógica de produção teórica, ou seja, não há distinção entre a prática da revisão de literatura e a prática de formulação de um quadro teórico (sucessão de citações sem encadeamento lógico e sem o estabelecimento de relações entre conceitos ou afirmações teóricas). Outra limitação ressaltada por Lima (1999) em relação aos referenciais teóricos dos estudos refere-se à ausência de distinção entre os textos empregados para construir a abordagem teórica empregada no estudo e os textos empregados para apresentar o conhecimento anterior existente sobre o problema estudado.

Além do rigor metodológico, os estudos precisam apresentar contribuições relevantes para a área de administração, como já destacado no estudo de Clark, Floyd e Wright (2006). Quanto à qualidade dos artigos científicos, Mason e Cox (1989) apontam que esta também depende da relevância do artigo para as comunidades acadêmica e profissional.

Como já referido neste ensaio teórico, a administração consiste em uma ciência aplicada (THOMSON, 1956), ou seja, procura atuar na resolução de problemas das organizações. Nesse sentido, a teoria administrativa não só reflete a prática organizacional, mas também ajuda a constituir essa prática (MARSDEN; TOWNLEY, 1996). 
A concepção da administração como ciência aplicada está em consonância com um dos tipos de pesquisa em administração destacado por Whitley (1982). Para o autor, existem dois tipos de pesquisa em administração. $O$ primeiro é similar às demais ciências sociais e se preocupa em entender e explicar por que os eventos e as ações aconteceram de tal modo. Essa compreensão ocorre por meio da identificação dos processos sociais subjacentes. Já o segundo tipo, que é focado na prática, busca não só entender o motivo pelo qual ocorreu o fenômeno, mas também intervir de forma a melhorar a situação. Assim, as pesquisas desenvolvidas na área necessitam demonstrar habilidade para afetar práticas sociais com êxito e promover a melhoria de práticas administrativas. Nesta direção, para que a administração tenha cientificidade, tanto precisa explicar os eventos e as ações, quanto melhorar, ao mesmo tempo, as práticas administrativas.

Em se tratando, ainda, da concepção da administração como ciência aplicada, Lima (1999) assegura que a produção científica traz, em si própria, uma busca pela aplicabilidade dos resultados obtidos, o que faz com que tal produção tenha a tendência de ser mais calcada na perspectiva de aplicação, como uma conexão imediata entre ciência e tecnologia aplicada.

Não obstante a importância atribuída à aplicabilidade da administração, Bertero e Keinert (1994) asseveram que, no Brasil, a produção acadêmica em estudos organizacionais possui um viés mais acadêmico com menor preocupação quanto à aplicabilidade prática e menor atenção às necessidades dos administradores nas organizações.

Percebe-se, assim, a relevância das pesquisas realizadas em administração e que esta relevância está amplamente relacionada ao fato de que a administração consiste em uma ciência aplicada (THOMPSON, 1956). Assim sendo, seus conhecimentos necessitam ser aplicáveis e relevantes às organizações, como também capazes de provocar alguma mudança nestas. Já que o objeto da administração consiste no estudo e na melhoria da coordenação e do controle de atividades humanas associadas (WHITLEY, 1977), em que a coordenação referese à gestão, e as atividades humanas associadas, às organizações, o seu foco necessita ser sempre a aplicabilidade. Dessa forma, além de se preocupar como o rigor metodológico, as pesquisas em administração devem se preocupar em apresentar contribuições aplicáveis e relevantes às organizações, de maneira a promover melhorias em sua gestão.

\section{CONSIDERAÇÕES FINAIS}

Neste ensaio, discutiu-se a dificuldade que a administração possui para atingir os critérios de cientificidade baseados nas ciências naturais, o que ocorre em virtude de seu objeto de estudo ser complexo e apresentar características das áreas sociais.

Assim, feita a constatação de que as ciências sociais e as naturais são diferentes, resta a pergunta: Qual delas é melhor? Talvez a grande maioria das pessoas e mesmo muitos pesquisadores responderiam que são as ciências naturais, por sua lógica de observação seguir a lógica matemática, garantindo a tão decantada objetividade científica. Contudo, percebe-se que esses critérios utilizados para comparar as ciências sociais e as naturais e, conseqüentemente, apresentar uma suposta "superioridade" das últimas em relação às primeiras, baseiam-se, exclusivamente, nos critérios das próprias ciências naturais. Diante disso, pergunta-se: se esses critérios são das ciências naturais, por que utilizá-los para julgar as ciências sociais? Embora essa utilização pareça deslocada, é o que acontece, além de que muitos estudiosos nem percebam que isso ocorra.

Então, por que são utilizados esses critérios? Simples: porque as ciências naturais são estudadas há muito mais tempo que as sociais e, portanto, foram usadas como modelo para julgar as ciências sociais. Sob esta ótica, questiona-se se é adequado utilizar uma ciência tão diferente para julgar a outra. Na opinião de Ramos (1989), por exemplo, os métodos e as técnicas das ciências naturais não devem ser considerados modelos de verdade. Diante do 
exposto, argumenta-se: se, como aponta Machlup (1994, p. 14), “os cientistas das ciências naturais normalmente não tentam fazer o que eles sabem que não podem fazer; e ninguém espera que eles o façam", por que se espera, portanto, que as ciências sociais realizem o impossível diante do objeto estudado?

Acredita-se que o problema resida exatamente na comparação de uma ciência com a outra. As ciências sociais e a administração podem ser inferiores em invariabilidade, porém são superiores em explicar os motivos pelos quais os fenômenos ocorrem. Assim, tudo depende do que está sendo valorizado; e o que costuma ser valorizado é o modelo das ciências naturais. Mas, se elas são diferentes, não deveriam ser valorizadas de forma diferente? Se, ao contrário, as ciências naturais fossem julgadas de acordo com o modelo das ciências sociais, se poderia dizer que as últimas são superiores, já que conseguem explicar os motivos pelos quais os fenômenos ocorrem. Mas esse não é o objetivo; o que se quer mostrar é que elas são diferentes e que, por isto, deveriam ser valorizadas de modos diferentes, levando em consideração as características e o contexto de cada uma.

Vale destacar, novamente, que ciências naturais e ciências sociais estudam fenômenos diferentes e têm objetivos diferentes. Costumam-se comparar objetos diferentes, como um computador e um automóvel, para dizer que o primeiro é melhor porque, com ele, se podem editar textos e fazer análises, se o último também pode ser melhor porque pode ser utilizado para se locomover? Bem, se não se costuma comparar um computador a um automóvel para dizer qual é o melhor, por que, então, comparar ciências sociais e ciências naturais?

O fato é que não está em questão encontrar qual é a melhor para descartar a pior. Se fosse assim, se compararia uma máquina de datilografar e um computador em relação à digitação de texto e se ficaria com o último por ser mais eficiente. O problema é que se está comparando o computador a um automóvel e, assim, um não pode substituir o outro. Se as ciências naturais pudessem estudar plenamente os fenômenos sociais e as organizações, aí, sim, se poderia, se comprovada sua "superioridade", descartar as ciências sociais e a administração. Contudo, isso não ocorre. Assim como as ciências sociais não buscam explicar os fenômenos naturais, as ciências naturais também não se propõem a explicar os fenômenos sociais. Portanto, uma não pode substituir a outra: ambas são extremamente importantes, pois somente elas explicam o seu próprio objeto de estudo.

Diante do fato de não se poder alterar o objeto de estudo da administração, ou seja, o estudo e a melhoria da coordenação e do controle de atividades humanas associadas (WHITLEY, 1977), e desconsiderada a possibilidade de comparar ciências sociais e ciências naturais em virtude de suas diferenças, resta, agora, considerar a segunda possibilidade: ampliação do rigor metodológico dos estudos em administração.

Sabe-se que há, na administração, a ausência de um consenso metodológico. Essa característica está relacionada à multidisciplinaridade da administração, visto que ela interage com o ambiente e se utiliza do conhecimento de outras áreas, como a psicologia, a sociologia, a economia, a ciência política e a antropologia (SANTOS, 2003), processo este que acaba por ocasionar um pluralismo metodológico na área.

A característica apontada permite, porém, múltiplas possibilidades de pesquisa, o que pode ser considerado uma vantagem para a administração. Percebe-se, em muitos estudos, a utilização, de forma conjunta ou complementar, de métodos qualitativos e de métodos quantitativos de pesquisa convergindo para um único objetivo. Se a administração tem conseguido gerar muitos conhecimentos e se valer desse pluralismo metodológico como uma vantagem, cabe a pergunta: Será que o caminho para um campo se consolidar como ciência é apresentar um único método? Ou apresentar métodos distintos que possam ser utilizados em fenômenos, também distintos, e, além disso, ainda combiná-los? Se tanto os métodos indutivos e dedutivos quanto os empíricos e racionais possibilitam a análise de fenômenos, bem como a elaboração e testes de teorias, e se nenhum método ou teoria é uma resposta 
absoluta, por que se necessitaria de um único método para atribuir cientificidade à administração?

Nesse contexto, defende-se que a administração não necessita de um método único, mas sim adotar maior rigor metodológico para garantir a validade dos conhecimentos gerados. Assim, o caminho para a administração se consolidar como uma ciência pode não ser o de procurar um método único ou empregar a lógica das ciências naturais, mas adotar um rigor metodológico que garanta a validade dos resultados, respeitando a complexidade do objeto estudado. Além disso, necessita-se buscar resultados que sejam relevantes às organizações no sentido de melhorar seus processos de gestão.

Apesar da importância do rigor metodológico, estudos apontam que as pesquisas em administração ainda têm muito a evoluir no que concerne aos aspectos metodológicos (STRAUB; CARLSON, 1989; HOPPEN; MEIRELLES, 2005; WALTER; BAPTISTA; AUGUSTO, 2008; CLARK; FLOYD; WRIGHT, 2006). Essa necessidade de evolução pode estar relacionada à classificação da administração como ciência em desenvolvimento (THOMPSON, 1956; BERTERO; CALDAS; WOOD JR., 1999), necessitando de maior amadurecimento.

O amadurecimento mencionado, considerando a realidade brasileira, já vem sendo buscado, se for levado em conta o aumento no número de programas de pós-graduação stricto sensu em administração, sendo, hoje, de 51 mestrados, 22 mestrados profissionais e 16 doutorados (CAPES, 2008b); o número de eventos Qualis para a administração, sendo 13 Nacionais "A” e 18 Nacionais “B”; e periódicos da área Qualis para a administração, sendo 73 Nacionais “A”, 37 Nacionais “B” e 24 Nacionais “C” (CAPES, 2008a), bem como que, com o sistema de avaliação da CAPES, professores e pesquisadores têm procurado obter uma pontuação minimamente adequada por meio da publicação de artigos.

Neste ensaio, apontaram-se duas razões pelas quais a administração encontra dificuldades para se consolidar como ciência. O primeiro motivo refere-se ao fato de a administração, como uma ciência social, não conseguir atingir alguns dos critérios de cientificidade das ciências naturais. Destaca-se, neste sentido, que os fenômenos sociais não são tão variáveis quanto podem parecer, porque, se não existissem regularidades, não seria possível nomear os fenômenos (MACHLUP, 1994) nem ensinar e treinar as pessoas para funções administrativas (THOMPSON, 1956).

A dificuldade de verificar hipóteses atinge as ciências sociais porque elas não podem isolar seu objeto de estudo em um laboratório. Não obstante, essa dificuldade também atinge as ciências naturais. A subjetividade, a falta de exatidão nos resultados, a dificuldade de prever eventos futuros e a relação com a história são outros exemplos de critérios que do mesmo modo atingem as ciências naturais (MACHLUP, 1994).

A mensurabilidade dos fenômenos costuma ser associada à quantificação, o que igualmente não pode ser atingido por todas as ciências naturais. Ainda sobre esse critério, é possível questionar se a quantificação consiste realmente no melhor indicador de mensurabilidade para a administração. Outro critério que deve ter sua importância questionada no que se refere à administração consiste no método homogêneo, visto que a heterogeneidade parece ser muito mais vantajosa para essa ciência.

Assim, percebe-se que tais critérios de cientificidade não deveriam ser o foco de preocupação da administração, uma vez que refletem falta de conhecimento e preconceito contra as ciências sociais; consistem, na realidade, em características das ciências sociais; afetam várias ciências, inclusive naturais; ou não são vantajosos ou adequados para a administração. Além disso, sendo as ciências sociais e as ciências naturais distintas, e não sendo possível substituir uma pela outra, torna-se incongruente julgar a administração sob os critérios das ciências naturais. 
A segunda razão destacada neste estudo para a dúvida de alguns membros da academia em relação à conceitualização da administração como ciência refere-se à validade e à relevância dos resultados obtidos pelos estudos em administração. No que tange a esses critérios, pesquisas como as de Straub e Carlson (1989), Clark, Floyd e Wright (2006), Hoppen e Meirelles (2005), Walter, Baptista e Augusto (2008), Bertero, Caldas e Wood Jr. (1999), Bertero e Keinert (1994) revelam que os estudos em administração necessitam aperfeiçoar vários aspectos metodológicos e de relevância. Dentre esses aspectos, destaca-se a importância de adotar procedimentos metodológicos rigorosos, independente do tipo de estudo realizado ou da posição epistemológica adotada; de esses estudos descreverem de forma detalhada os procedimentos adotados em relação ao desenho de pesquisa, à coleta e à análise de dados e à validação da pesquisa; de uma revisão de literatura que configure a lógica de produção teórica e que distinga textos empregados para construir a abordagem teórica e os empregados para apresentar o conhecimento anterior; da aplicabilidade prática e da atenção às necessidades dos administradores nas organizações; e da contribuição teórica e prática dos estudos.

Neste sentido, observa-se que as pesquisas em administração necessitam ampliar seu rigor metodológico e apresentar contribuições importantes e práticas para a gestão das organizações, visto que, com a conduta metodológica atualmente empregada em parte dos estudos administrativos, a administração terá dificuldades de gerar conhecimentos válidos capazes de alterar práticas cotidianas das organizações.

Assim, respondendo à pergunta de pesquisa adotada neste estudo, argumenta-se que a administração, para ser considerada uma ciência, não deve buscar atender aos critérios de cientificidade das ciências naturais, mas, sim, focar seus esforços para produzir pesquisas com maior validade e relevância para sua área conhecimento.

Diante dos aspectos apontados e discutidos neste ensaio, reafirma-se a posição de que a administração pode gerar conhecimento científico, podendo ser considerada uma ciência. Contudo, necessita, independente da posição epistemológica, zelar mais pelo rigor metodológico dos seus estudos, para que os resultados obtidos sejam válidos, bem como para que as contribuições sejam relevantes e melhorem a gestão das organizações, visto que a administração consiste em uma ciência aplicada.

\section{REFERÊNCIAS}

ASTLEY, W. Graham. Administrative science as socially constructed truth. Administrative Science Quarterly, n. 30, p. 497-513, 1985.

BAGOZZI, Richard P. Causal modeling in marketing. New York: Wiley \& Sons, 1980.

BASSO, Maximino. Ciências humanas e ciências naturais, na visão de Boaventura Santos. Humanitates. v. 1, n. 2. Nov. 2004.

BERTERO, Carlos O; CALDAS, Miguel P; WOOD JR, Thomaz. Produção científica em administração de empresas: provocações, insinuações e contribuições para um debate local. Revista de Administração Contemporânea, v. 3, n. 1, p. 147-178, jan./abr.1999.

BERTERO, Carlos O; KEINERT, Tania M. M. A Evolução da Análise Organizacional no Brasil. Revista de Administração de Empresas, v. 34, n. 3, p. 81-90, 1994.

BREWER, John; HUNTER, Albert. Foundations of multimethod research. Thousand Oaks: Sage, 2006. 
BURREL, Gibson; MORGAN, Gareth. Sociological paradigms and organizational analysis: elements of the sociology of corporate life. Londres: Ashgate, 2000.

CAPES - Coordenação de Aperfeiçoamento de Pessoas de Nível Superior. Classificação de periódicos, anais, revistas e jornais. Disponível em: $<$ http://qualis.capes.gov.br/webqualis/>. Acesso em: 15 maio 2008a.

Relação de Cursos Recomendados e Reconhecidos. Disponível em: $<$ http://servicos.capes.gov.br/projetorelacaocursos/jsp/grandeAreaDet.jsp>. Acesso em: 15 maio 2008b.

CLARK, Timothy; FLOYD, Steven W; WRIGHT, Mike. On the Review Process and Journal Development. Journal of Management Studies, v. 43, n. 3, maio 2006, p. 655-664.

DIJK, Nic van; PUNCH, Maurice. Useful Knowledge. Management Science as Dialogue and Confrontation, 1993.

GRANGER, Gilles-Gaston. A ciência e as ciências. São Paulo: UNESP, 1994.

HABERMAS, Jürgem. Técnica e ciência como ideologia. Lisboa: Edições 70, 1968.

HATCH, Mary Jo; YANOW, Dvora. Organization theory as an interpretive science. In: TSOUKAS, Haridimos; KNUDSEN, Christian (org.). The Orford Handbook of Organization Theory: meta-theoretical perspectives. 2. ed. Nova York: Oxford, 2005.

HOPPEN, Norberto; MEIRELLES, Fernando S. Sistemas de informação: um panorama da pesquisa científica entre 1990 e 2003. Revista de Administração de Empresas, v. 45, n. 1, jan./mar. 2005, p. 24-35.

LÉVI-STRAUSS, Claude. Antropologia estrutural II. Rio de Janeiro: Tempo Brasileiro, 1976.

LIMA, Juvêncio B. de. Pesquisa qualitativa e qualidade na produção científica em administração de empresas. In: ENCONTRO ANUAL DA ASSOCIAÇÃO NACIONAL DE PÓS-GRADUAÇÃ̃ E PESQUISA EM ADMINISTRAÇÃO, 23, 1999, Foz do Iguaçu. Anais... Foz do Iguaçu: ANPAD, 1999, p. 1-15.

LOPES, Alice R. C; MACEDO, Elizabeth F. de. Das ciências naturais às ciências sociais: o currículo segundo William Doll. Educação On-Line. 2001.

MACHADO-DA-SILVA, Clóvis L; CUNHA, Vera C. Da; AMBONI, Nério. Organizações: o estado da arte da produção acadêmica no Brasil. In: ENCONTRO ANUAL DA ASSOCIAÇÃO NACIONAL DE PÓS-GRADUAÇÃO E PESQUISA EM ADMINISTRAÇÃO, 14, 1990, Florianópolis. Anais... Florianópolis: ANPAD, 1990. v. 6. p. $11-28$.

MACHLUP, Fritz. Are the social sciences really inferior? In: MARTIN, Michael; McINTYRE Lee C. Readings in the Philosophy of Social Science. Cambridge: The MIT Press, 1994, p. 5-20. 
MARSDEN, Richard; TOWNLEY, Bárbara. The owl of Minerva: reflections on theory in practice. In: CLEGG, Steward; HARDY, Cynthia; NORD, Walter R. Handbook of Organization Studies. London, Sage, 1996.

MASON, R. O; COX, E. L. MIS experiments: a pragmatic perspective. In: BENBASAT, I. (ed.). The Information Systems Research Challenge: Experimental Research Methods. Harvard Business School Research Colloquium, v. 2. Boston, MA: Harvard Business School, 1989. p. 3-29.

POPPER, Karl R. Conhecimento objetivo: uma abordagem evolucionária. Belo Horizonte: Itatiaia; São Paulo: Universidade de São Paulo, 1975.

RAMOS, Alberto G. A nova ciência das organizações: uma reconceituação da riqueza das nações. 2. ed. Rio de Janeiro: Fundação Getúlio Vargas, 1989.

SANTOS, Silvio A. dos. Existem Paradigmas em Administração? Uma análise sobre o uso do conceito. In: INTERNATIONAL CONFERENCE IBEROAMERICAN ACADEMY OF MANAGEMENT, 3, 2003, São Paulo. Anais..., 2003. p. 1-20.

SCHUTZ, Alfred. Fenomenologia e relações sociais. Rio de Janeiro: Zahar, 1979.

SILVA, Eduardo D. Por que la administración de empresas es una ciencia?. In: CONGRESSO LATINO-AMERICANO DE ESCOLAS DE ADMINISTRAÇÃO, 37, 2002, Porto Alegre. Anais..., 2002.

STRAUB, Detmar W; CARLSON, Curtis L. Validating instruments in MIS research. MIS Quarterly, v. 13, n. 2, jun. 1989.

THOMPSON, James D. On building an administrative science. Administrative Science Quarterly, v. 1, n. 1, p. 102-111, 1956.

TSOUKAS, Haridimos; CUMMINGS, Stephen. Marginalization and recovery: the emergence of Aristotelian themes in Organization Studies. In: Organization Studies, v. 18, n. 4, 1997.

WALTER, Silvana A; BAPTISTA, Paulo de P; AUGUSTO, Paulo O. M. Visão baseada em recursos: uma análise dos delineamentos metodológicos e da maturidade dessa abordagem na área de estratégia do EnANPAD 1997-2007. In: ENCONTRO ANUAL DA ASSOCIAÇÃO NACIONAL DE PÓS-GRADUAÇÃO E PESQUISA EM ADMINISTRAÇÃO, 32, 2008, Rio de Janeiro. Anais... Rio de Janeiro: ANPAD, 2008. p. 1-16.

WEBER, Max. The theory of social and economic organization. Nova Iorque: Oxford Univerty Press, 1947.

WHITLEY, Richard. The establishment and structure of the sciences as reputational organizations. In: ELIAS, N; MARTINS, H; WHITLEY, R. (eds.). Scientific establishments and hierarchies. p. 313-357. Dordrecht, Holland: D. Reidel, 1982.

The scientific status of management research as pratically-oriented social science.

Journal of Management Studies, v. 21, n. 4,1984. 
The sociology of scientific work and the history of scientific developments. In: BLUME, Stuart S. (ed.). Perspectives in the Sociology of Science, John Wiley and Sons, Chichester-New York-Brisbane-Toronto, p. 21-50. 1977 